aluminium, and Jayaraman, Klement and Kennedy ${ }^{6}$ that of germanium and of silicon. Pressure was inferred from piston force and area, with a correction for piston friction, giving an accuracy of $1.0 \mathrm{kbar}$.

Fig. 2 shows a very satisfactory agreement between the fusion curves obtained in the present work and a linear extrapolation of those of Butuzov ${ }^{3}$ for zinc and aluminium: further, that, in the range up to $60 \mathrm{kbar}$ and $1,100^{\circ} \mathrm{C}$, reasonable agreement is also obtained with the curves obtained in refs. 4 and 6 for zinc, silicon and germanium. Considering that the various determinations were made in widely differing types of apparatus, and in particular that Butuzov's conditions were truly hydrostatic, it is a reasonable conclusion that the effect of temperature on the load/pressure calibration curve for the tetrahedral apparatus is small, certainly less than $\pm 3 \mathrm{kbar}$ at $50 \mathrm{kbar}$ and $1,000^{\circ} \mathrm{C}$.

There is a significant difference, amounting to $8 \mathrm{kbar}$ (or $50^{\circ} \mathrm{C}$ ) at $40 \mathrm{kbar}$ and $900^{\circ} \mathrm{C}$, between the present results for aluminium and those of ref. 5. It is possible that at least some of this effect is due to the reaction discussed above between molten aluminium and the molybdenum containers used in the latter work.

In a recent determination of the phase diagrams of silicon and germanium, Bundy ${ }^{7}$ used a modified 'belt' type of apparatus to produce pressures up to about $200 \mathrm{kbar}$ and $1,000^{\circ} \mathrm{C}$. With this apparatus a large pressure rise due to local heating at the specimen was inferred, which was roughly proportional to the product of the initial pressure and the temperature rise, and would amount to about $16 \mathrm{kbar}$ at $50 \mathrm{kbar}$ and $1,000^{\circ} \mathrm{C}$. The compatibility of the present results with those of other workers implies that such a large effect is not encountered with the tetrahedral apparatus as used in this work.

We thank Miss A. Thake, who carried out many of the experiments, and L. Hailes for the microprobe analysis of specimens; we also thank C. H. L. Goodman for discussion.

J. LeES

B. H. J. Williamson

Standard Telecommunication Laboratories, Ltd., Harlow, Essex.

${ }^{1}$ Modern Very High Pressure Techniques, edit. by Wentorf, R. H., 1 (Butterworths, London, 1962).

¿ Lees, J., Nature, 203, 965 (1964).

${ }^{3}$ Butuzov, V. P., Sov. Phys. Crystallogr., 2, 533 (1957).

${ }^{4}$ Kennedy, G. C., and Newton, R. C., in Solids Under Pressure, edit. by Paul, W., and Warschauer, D. M., 163 (MeGraw-Hill, New York, 1963).

$\checkmark$ Jayaraman, A., Klement, W., Newton, R. C., and Kennedy, G. C., Intern. J. Phys. Chem. Solids, 24, 7 (1963).

- Jayaraman, A., Klement, W., and Kennedy, G. C., Phys. Rev., 130, 540 (1963). 'Bundy, F. P., J. Chem. Phys., 41, 3809 (1964).

\section{A New Absolute Determination of the Acceleration due to Gravity at the National Physical Laboratory}

An absolute determination of the acceleration due to gravity at a site in the National Physical Laboratory has recently been completed. It is the first to be made by timing the symmetrical up-and-down motion of a body moving freely under gravity, and the uncertainty of the result is considerably less than that of any previous measurement.

If a body is timed as it crosses two horizontal planes with a vertical separation $H$, and if the time interval between crossings of the lower plane is $T_{1}$ and of the upper plane $T_{2}$, then:

$$
g=\frac{8 H}{T_{1}^{2}-T^{2}}
$$

A correction has to be applied for the decrease of gravity with height.

In the present experiment the planes were defined optically by pairs of horizontal slits and the moving object was a glass ball which focused one slit of a pair on the other when it was symmetrically between them. One slit was illuminated and a photomultiplier behind the other recorded the flash of light as the ball passed. The pairs of slits were incorporated in composite blocks of fused silica, the vertical distance $(1 \mathrm{~m})$ between the blocks at the upper and lower positions being measured by means of an interferometer.

The advantages of this method have been discussed recently ${ }^{x}$ and it is sufficient to say that they have been fully borne out; in particular the measured acceleration is independent of air resistance.

The measured value of gravity, reduced to the British Fundamental Station in the National Physical Laboratory $^{2}$, is 981181.77 mgal, with a standard deviation of $0 \cdot 13 \mathrm{mgal}\left(1 \mathrm{mgal}=10^{-5} \mathrm{~m} / \mathrm{sec}^{2}\right)$.

The uncertainty comes mainly from microseismic disturbances. An earlier determination at the National Physical Laboratory with a reversible pendulum ${ }^{3}$ gave a value of $981183 \cdot 2$, s.d. $0 \cdot 7 \mathrm{mgal}$ at the British Fundamental Station. The difference of 1.4 mgal from the new result is just twice the standard deviation of Clark's value. Comparisons with other determinations will be discussed in the full account of the measurements which will be published later.

Standards Division,

A. H. $\mathrm{CoOK}$

National Physical Laboratory,

Teddington, Middlesex.

1 Cook, A. H., Metrologia, 1, 84 (1965).

The British Fundamental Gravity Station, Nature, 173, 794 (1954).

${ }^{3}$ Clark, J. S., Phil. Trans. Roy. Soc., 238, 65 (1939); Jeffreys, H., Mon. Not. Roy. Astron. Soc., Geophys. Supp., 5, 398 (1949).

\section{Deformation of Gas Bubbles and Liquid Drops in an Electrically Stressed Insulating Liquid}

THE recent paper by Garton and $\mathrm{Krasucki}^{1}$ is of considerable interest and reminded me of some of the work I undertook about five years ago ${ }^{2}$. I observed a similar phenomenon under different conditions. This brief note is to bring to notice some of my results obtained at that time, which serve as further evidence that gas bubbles and liquid drops always tend to elongate in the direction of the applied field in an insulating liquid, irrespective of whether the permittivity of the bubble or drop is larger or smaller than that of the surrounding liquid.

A glass test cell 5 in. $\times 3$ in. $\times 2$ in. was used, contain. ing a pair of parallel brass plane electrodes of size $4 \mathrm{in.} \times$ $2 \mathrm{in}$. with a separation of $0.5 \mathrm{in}$. aligned horizontally. The test cell was fully filled with transformer oil, and an air bubble or a water drop could be introduced into the cell through two small holes in the top and the bottom of the cell. The pressure on the liquid was kept at atmospheric pressure and the temperature $20^{\circ} \mathrm{C}$. The air bubble was injected through the bottom hole and it moved up through the centre of the liquid gap by buoyancy force, while the water drop was injected through the top hole and it moved down through the centre of the liquid gap by gravitation force. The $50-\mathrm{c} / \mathrm{s}$ a.c. field was first applied and a photograph was taken when the bubble reached the centre of the liquid gap. This method was different from that adopted by Garton and Krasucki, who deliberately eliminated the buoyancy force by rotating the test cell to maintain the bubble in a fixed position. However, their results and mine agree qualitatively. Fig. 1 shows an air bubble elongated at $22.5 \mathrm{kV}$ and $28.5 \mathrm{kV}$ though they were slightly distorted. Fig. 2 shows a water drop elongated and a large drop bursting into many droplets at $6 \mathrm{kV}$. The bubbles and drops in $(a),(b)$ and $(c)$ of Figs. 1 and 2 were not originally idontical in size, since the device could not control their sizes accurately, But it can be scen that the greater the difference in permittivity between the bubble (or drop) and the insulating liquid, and the larger the original size of the bubble (or drop), the smaller is the electrical field required to cause elongation-as predicted by theor $y^{1,2}$. 